\title{
Elastic and inelastic cross sections calculated within the CDCC approach for deuteron induced reactions
}

\author{
P. Chau Huu-Tai ${ }^{\mathrm{a}}$
}

CEA/DIF, BP. 12, 91680 Bruyères-le-Châtel, France

\begin{abstract}
The Continuum Discretized Coupled Channels (CDCC) equations have been extended to rotational nuclei. Within this approach, the deuteron breakup channels and the target excitations are treated simultaneously and one can calculate both the elastic and the inelastic cross sections. Comparisons of the calculated elastic and inelastic differential cross sections with experimental data have been performed for deuteron incident on ${ }^{116} \mathrm{Sn},{ }^{90} \mathrm{Zr}$ at $183 \mathrm{MeV}$ and on ${ }^{70} \mathrm{Ge},{ }^{72} \mathrm{Ge},{ }^{24} \mathrm{Mg},{ }^{16} \mathrm{O}$ at $171 \mathrm{MeV}$. Calculations have also been done for ${ }^{24} \mathrm{Mg}$ and ${ }^{12} \mathrm{C}$ targets for incident energies between 60 and $90 \mathrm{MeV}$. A satisfactory overall agreement has been obtained.
\end{abstract}

\section{Introduction}

Breakup channels can play a crucial role when dealing with reaction mechanism involving weakly bound projectile. The Continuum Discretized Coupled Channels (CDCC) method $[1,2]$ is a three-body problem approximation which has been developped to take explicitly into account these breakup channels. Initially proposed to describe deuteron induced reactions, it has been widely studied from a theorical point of view and quite successfully compared with experimental data (see e.g., [3-6]). It has been more recently used and extended to describe reactions involving halo nuclei improving our knowledge of these exotic systems (see e.g., [7] and references therein). Nevertheless, as far as we know, few calculations involving both the projectile breakup and the target excitations have be performed [5]. Therefore I propose in this paper to present some recent results where the target excitations and the projectile breakup are treated on the same footing for deuteron induced reactions. This contribution is organized as follows. In the second section, I will briefly remind the formalism and hypotheses. The third section is devoted to the comparison with experimental data.

\section{Formalism and hypotheses}

I have assumed that

- the target spectrum is a rotational one;

- the deuteron and proton-neutron continuum model space can be limited to ${ }_{1}^{3} S$ and ${ }_{1}^{3} D$ waves;

- the continuum can be discretized by the means of 4 bins;

- no closed channel has been used to describe the continuum.

The wave function of the system $\left|\Phi_{J_{T} M_{T}}\right\rangle$ with total angular momentum $J_{T}$ and projection $M_{T}$ along the z-axis can be written as

$$
\begin{aligned}
\left|\Phi_{J_{T} M_{T}}(\boldsymbol{\rho}, \boldsymbol{R})\right\rangle & =\sum_{i l S I_{p} L J I_{t}} U_{c}(R) i^{L} \\
& \times\left[\left[\left[\varphi_{i l}(\boldsymbol{\rho}) \otimes \chi_{S}\right]^{I_{p}} \otimes Y_{L}(\hat{R})\right]^{J} \otimes \psi_{I_{t}}\left(\xi_{t}\right)\right]_{M_{T}}^{J_{T}}
\end{aligned}
$$

where $\boldsymbol{\rho}, \boldsymbol{R}$ and $\xi_{t}$ represent the proton-neutron relative coordinates, the proton-neutron center of mass ones and the target variables, respectively. In equation (1), $c$ denotes the channel labeled by ( $\left.i l S I_{p} L J I_{t}\right)$ where

- $i$ is the bin number for the proton-neutron wave function $(0 \leq i \leq 4)$;

- $l$ denotes the orbital angular momentum of the relative motion between the proton and the neutron: $l=0$ or 2 ;

- $S=1$ is the spin of the proton-neutron;

- $I_{p}$ is obtained by coupling $l$ and $S$;

- $L$ is the orbital angular momentum associated to the motion of the proton-neutron center of mass;

- $J$ is the coupling between $L$ and $I_{p}$;

$-I_{t}$ is the spin of the target level.

$\varphi_{i l}$ and $\psi_{I_{t}}$ represent the deuteron wave function and the target state.

The total hamiltonian of the system can be written as

$$
\hat{H}=\hat{T}_{R}+V_{p A}\left(\boldsymbol{r}_{p}, \xi_{t}\right)+V_{n A}\left(\boldsymbol{r}_{n}, \xi_{t}\right)+V_{C o u l}+\hat{H}_{p n}+\hat{H}_{A}
$$

with

$$
\hat{H}_{p n} \varphi_{i l}(\rho)=\varepsilon_{i} \varphi_{i l}(\rho) \text { and } \hat{H}_{A} \psi_{I_{t}}\left(\xi_{t}\right)=\hat{\varepsilon}_{I_{t}} \psi_{I_{t}}\left(\xi_{t}\right)
$$

Following the work of Tamura [8], one finds that, for rotational nuclei, each optical potential $V_{a A}\left(\boldsymbol{r}_{a}, \xi_{t}\right)(a=n$ or $p$ ) can be expressed as

$$
V_{a A}\left(\boldsymbol{r}_{a}, \xi_{t}\right)=\sum_{\lambda \mu} \sqrt{\frac{4 \pi}{2 \lambda+1}} V_{a A}^{(\lambda)}\left(r_{a}\right) D_{\mu 0}^{\lambda}\left(\Theta_{k}\right) Y_{\lambda \mu}\left(\theta_{a}, \phi_{a}\right)
$$

where $\Theta_{k}(k=1,2,3)$ denote the Eulerian angles. In equation (4), $V_{a A}^{(\lambda)}$ depends only on the radial variable $r_{a}$ and on the deformation parameters of the target. The main difficulty stems from the fact that, in equation (4), the potential depends on the angular variables of the nucleon. This technical issue can be solved by using solid spherical harmonics and the appropriate addition theorem [9] thus the nucleon-target optical potential 


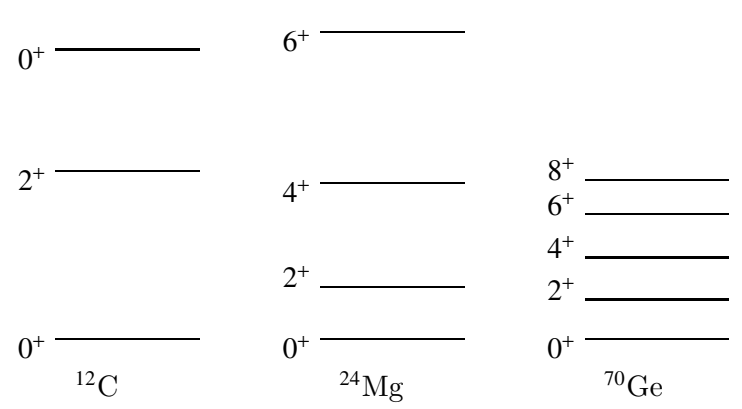

Fig. 1. Ground state bands for ${ }^{12} \mathrm{C},{ }^{24} \mathrm{Mg}$ and ${ }^{70} \mathrm{Ge}$ targets. Only the ${ }^{24} \mathrm{Mg}$ one exhibits rotational features.

can be rewritten as

$$
\begin{gathered}
V_{a A}\left(\boldsymbol{r}_{a}, \xi_{t}\right)=\sum_{\lambda \mu p} \frac{V_{a A}^{(\lambda)}\left(r_{a}\right) x_{a}^{p} R^{p} y_{a}^{\lambda-p} \rho^{\lambda-p}}{r_{a}^{\lambda}} D_{\mu 0}^{\lambda}\left(\Theta_{k}\right) \\
\sqrt{\frac{4 \pi(2 \lambda+1) !}{(2(\lambda-p)+1) !(2 p+1) !}}\left[Y^{p}(\hat{R}) \otimes Y^{\lambda-p}(\hat{\rho})\right]_{\mu}^{\lambda}
\end{gathered}
$$

where $\boldsymbol{r}_{a}=x_{a} \boldsymbol{R}+y_{a} \boldsymbol{\rho}$ and $0 \leq p \leq \lambda$.

One can then obtain the set of coupled differential equations

$$
\begin{gathered}
{\left[-\frac{\hbar^{2}}{2 \mu_{R}}\left(\frac{d^{2}}{d R^{2}}-\frac{L(L+1)}{R^{2}}\right)+F_{c c}(R)+V_{C o u l}(R)-E_{c}\right] U_{c}(R)} \\
=-\sum_{c^{\prime}} F_{c c^{\prime}}(R) U_{c^{\prime}}(R)
\end{gathered}
$$

with $E_{c}=E-\varepsilon_{i}-\hat{\varepsilon}_{I_{t}}$ and where the coupling $F_{c c^{\prime}}=$ $\left\langle i l S I_{p} L J I_{t} ; J_{T} M_{T}\left|V_{p A}+V_{n A}\right| i^{\prime} l^{\prime} S^{\prime} I_{p}^{\prime} L^{\prime} J^{\prime} I_{t}^{\prime} ; J_{T} M_{T}\right\rangle$ is derived using equation (5) and the Wigner-Eckart theorem. tions

The solutions must satisfy the following boundary condi-

$$
U_{c}(R) \rightarrow \delta_{c_{0} c} u_{L}^{-}\left(P_{c} R\right)+\sqrt{P_{c} / P_{c_{0}}} S_{c_{0} c} u_{L}^{+}\left(P_{c} R\right)
$$

where $c_{0}$ denotes the elastic channel and $u_{L}^{-}, u_{L}^{+}$are the incoming and outgoing Coulomb wave functions, respectively.

The $S$-matrix elements and the cross sections are calculated from these solutions.

\section{Comparison with experimental data}

All the calculations have been performed by using the KoningDelaroche parameterization for the nucleon-target optical potentials. The spin-orbit interaction has been set to zero. Only the first $2^{+}$excited level of the target has been taken into account.

\section{1 ${ }^{116} \mathrm{Sn},{ }^{90} \mathrm{Zr},{ }^{70} \mathrm{Ge},{ }^{72} \mathrm{Ge},{ }^{24} \mathrm{Mg}$ and ${ }^{16} \mathrm{O}$ targets at intermediate energies}

Baümer et al. [10] and Korff et al. [11] have studied the deuteron elastic and inelastic scattering on ${ }^{116} \mathrm{Sn},{ }^{90} \mathrm{Zr}$ at $183 \mathrm{MeV}$ and on ${ }^{70} \mathrm{Ge},{ }^{72} \mathrm{Ge},{ }^{24} \mathrm{Mg},{ }^{16} \mathrm{O}$ at $171 \mathrm{MeV}$. Even if many of these nuclei can not be considered as rotational
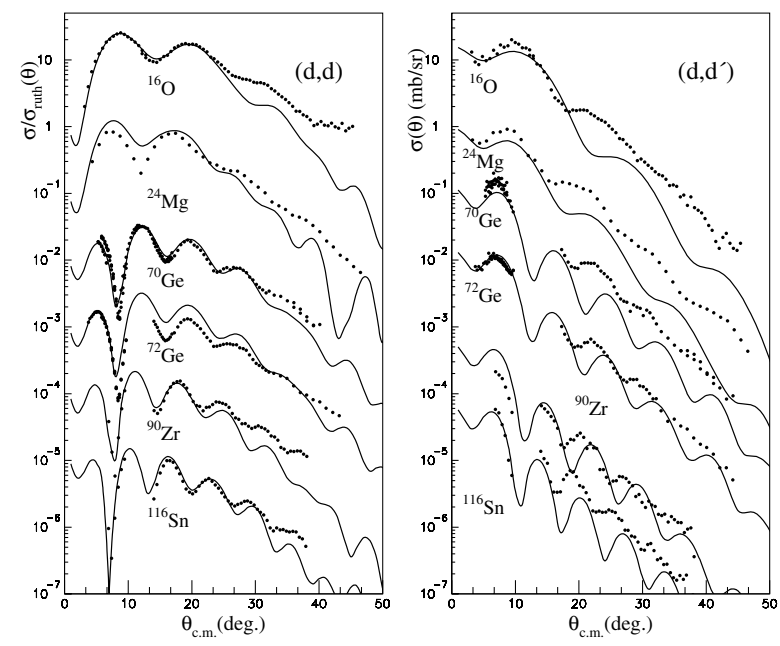

Fig. 2. Elastic and inelastic cross sections on the first $2^{+}$for deuteron incident on ${ }^{116} \mathrm{Sn},{ }^{90} \mathrm{Zr}$ at $183.0 \mathrm{MeV}$ and on ${ }^{70} \mathrm{Ge},{ }^{72} \mathrm{Ge},{ }^{24} \mathrm{Mg},{ }^{16} \mathrm{O}$ at 171.0 MeV [10,11]. The solid lines and the dots represent the CDCC calculations and the experimental data, respectively.

Table 1. $\beta_{2}$ deformation parameters.

\begin{tabular}{cccccccc}
\hline & ${ }^{116} \mathrm{Sn}$ & ${ }^{90} \mathrm{Zr}$ & ${ }^{70} \mathrm{Ge}$ & ${ }^{72} \mathrm{Ge}$ & ${ }^{24} \mathrm{Mg}$ & ${ }^{16} \mathrm{O}$ & ${ }^{12} \mathrm{C}$ \\
\hline$\beta_{2}$ & 0.2 & 0.2 & 0.2 & 0.2 & 0.4 & 0.2 & -0.47 \\
\hline
\end{tabular}

systems (some ground state bands are plotted in fig. 1). I have tried to analyse these data with the model presented in the previous section. The values of the deformation parameters used are summarized in table 1 . On the left part of figure 2, the ratios of the differential elastic cross sections to the Rutherford cross sections have been plotted. The solid lines and the dots represent the curves obtained by the CDCC calculations and the experimental data, respectively. The targets are indicated next to the curves. On the right part of this figure is drawn the inelastic cross sections on the first $2^{+}$. The CDCC results are plotted as solid lines and the dots represent the experimental data. The curves and the data points at the top represent true values, while the others are offset by factor 10, 100, etc. For the elastic cross sections, one observe quite a good agreement with the experimental data for both the amplitude and the diffraction patterns except for ${ }^{72} \mathrm{Ge}$. For this target, the amplitude of the calculated cross section becomes to large for angles larger than 15 degrees. A good agreement has also been obtained for the inelastic cross sections.

\section{$3.2{ }^{12} \mathrm{C}$ target}

In [12], Aspelund et al. have measured the elastic and inelastic deuteron scattering on ${ }^{12} \mathrm{C}$ target at $60.6,77.3$ and $90.0 \mathrm{MeV}$. They have analysed these data within a coupled channel approach using an ajusted optical potential and they concluded that the quadrupole deformation parameter $\beta_{2}$ had to be set to -0.48 to reproduce the inelastic cross sections. Hinterberger et al. have measured the elastic cross section at $52 \mathrm{MeV}$ [13] and Ishida et al. have measured the elastic and the inelastic cross sections at $53 \mathrm{MeV}$ [14]. Their results are depicted as full 

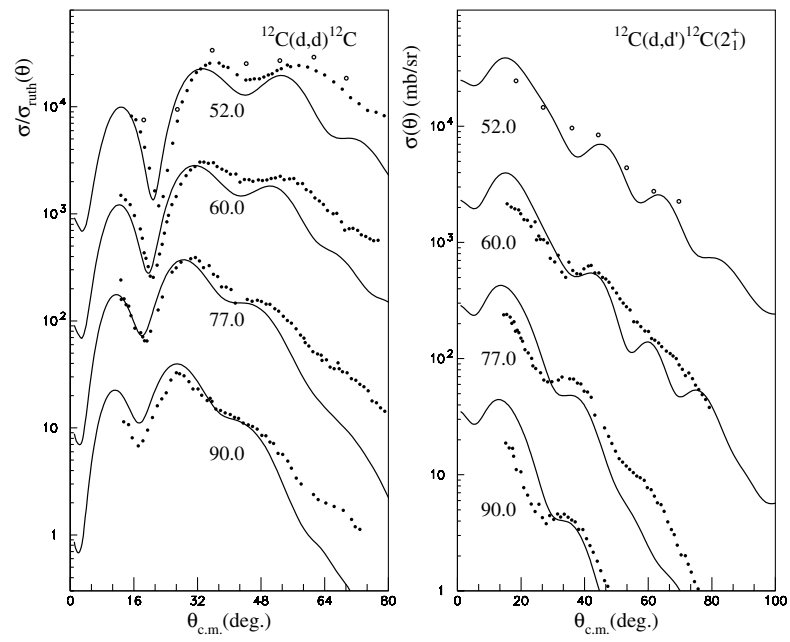

Fig. 3. Elastic and inelastic cross section for deuteron incident on ${ }^{12} \mathrm{C}$ target at 52 [13,14], 60, 77 and $90 \mathrm{Mev}$ [12]. The experimental data are represented by dots while the CDCC calculations are plotted with solid lines. The incident energies used in the calculations are indicated in $\mathrm{MeV}$ near the curves.

and open symbols in figure 3, respectively. I have performed calculations within the CDCC approach assuming that the quadrupole deformation parameter is -0.47 . The results are plotted in figure 3: the ratios of the differential elastic cross sections to the Rutherford cross sections and the inelastic cross sections have been plotted on the left part and the right one, respectively. The incident laboratory energies are indicated in $\mathrm{MeV}$ next to the curves.

From these curves one can note that

- Some discrepancies are observed at the four energies for the elastic cross sections: there is a slight phase shift between the experimental diffraction patterns and the calculated ones. At $90 \mathrm{MeV}$, the amplitudes are not very well reproduced.

- Quite a good agreement is obtained for the inelastic cross sections.

To explain these differences between the calculations and the experimetal data, one can firstly note that the adopted nucleontarget optical potential parameterization has been proposed for nuclides in the mass range $24 \leq \mathrm{A} \leq 209$. Thus one should not expect to find a perfect agreememt for the ${ }^{12} \mathrm{C}$ target. Then as seen in figure 1, the spectrum of this target is not a rotational one and the chosen model is certainly not the best one to describe the reaction mechanism and the target excitation. Despite these crude hypotheses, the experimental trends are quite well reproduced by the calculations and it should be emphasize that there is no free parameter in these CDCC calculations.

\section{$3.3{ }^{24} \mathrm{Mg}$ target}

Among all the nuclei considered in this paper, the ${ }^{24} \mathrm{Mg}$ target is almost the only one that can be seen as a rotational system. Furthermore a large set of experimental data is available since Kiss et al. [15] have performed study of elastic and inelastic deuteron scattering on ${ }^{24} \mathrm{Mg}$ for incident energies ranging
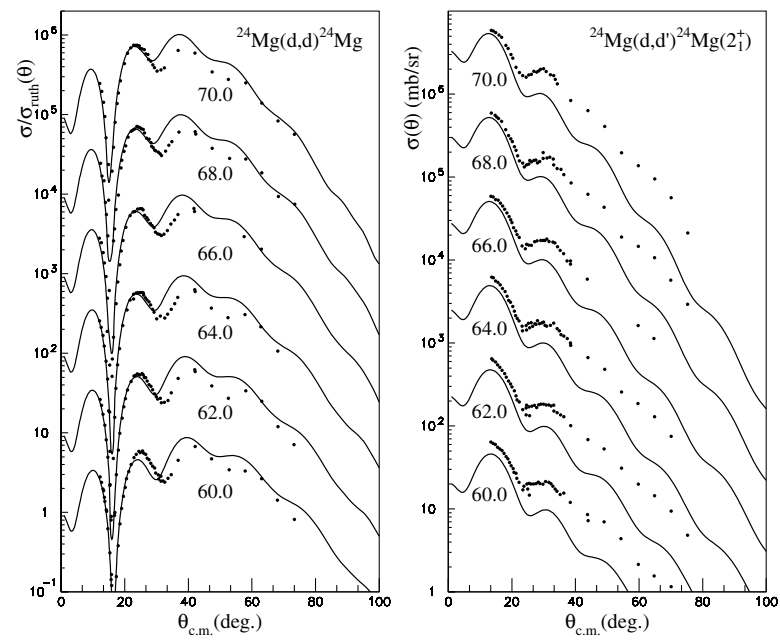

Fig. 4. Elastic and inelastic cross section for deuteron incident on ${ }^{24} \mathrm{Mg}$ target at 60, 62, 64, 66, 68 and $70 \mathrm{Mev}$ [15]. The experimental data are represented by dots and the CDCC calculations are plotted as solid lines. The incident energies are indicated in $\mathrm{MeV}$ near the curves.
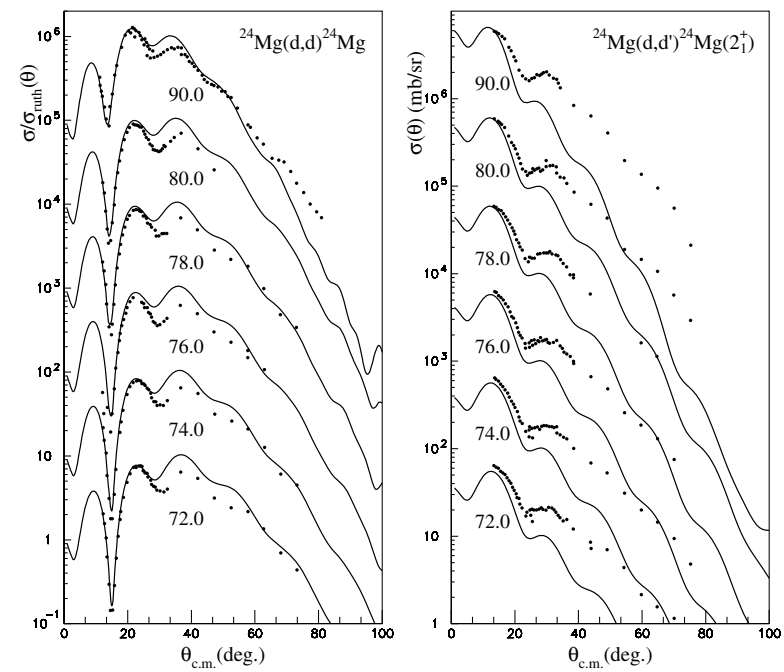

Fig. 5. Elastic and inelastic cross section for deuteron incident on ${ }^{24} \mathrm{Mg}$ target at 72, 74, 76, 78, 80 and $90 \mathrm{Mev}$ [15]. The experimental data are represented by dots while the CDCC calculations are plotted as solid lines. The incident energies are indicated in $\mathrm{MeV}$ near the curves.

from 60 to $90.0 \mathrm{MeV}$. Thus the comparison with these data provides a good test of the validity of the CDCC formalism. The experimental results are plotted as dots in figures 4 and 5 .

From the elastic cross sections, Kiss et al. have determined a set of parameters for a surface absorbing optical potential that gives the best fit of the data and with these parameters, they have deduced the quadrupole deformation parameter that gives the best agreement with the inelastic cross sections within the coupled channel framework. This value for $\beta_{2}$ is found to be 0.4 . I have used this parameter while building the optical potentials by folding of the nucleon-target potentials. The results are represented as solid lines in 4 and 5. One can note that there is a good agreement between the CDCC calculations and the experimental data for both the elastic and the inelastic cross sections. 


\section{Conclusion}

I have proposed an extension of the CDCC method for deuteron induced reaction which takes into account both the deuteron breakup and the target excitations for rotational nuclei. It has been applied to analyse some experimental data at intermediate energies and energies ranging from 60 to $90 \mathrm{MeV}$ for ${ }^{116} \mathrm{Sn},{ }^{90} \mathrm{Zr},{ }^{70} \mathrm{Ge},{ }^{72} \mathrm{Ge},{ }^{24} \mathrm{Mg},{ }^{16} \mathrm{O}$ and ${ }^{12} \mathrm{C}$ targets. Emphasizing the fact that there is no ajusted parameter, the agreement is quite good even if some improvement have to be achieved for Ge and $\mathrm{C}$ targets.

I would like to thank my colleagues of the Service de Physique Nucléaire. I also thank the exploitation staff of the Centre de Calcul Recherche et Technologie where the calculations have been partly performed.

\section{References}

1. R.C. Johnson, P.J.R. Soper, Phys. Rev. C 1, 976 (1970).

2. G.H. Rawitscher, Phys. Rev. C 9, 2210 (1974).
3. N. Austern et al., Phys. Rep. 154, 125 (1987).

4. M. Yahiro, M. Kamimura, Prog. Theor. Phys. 65, 2046 (1981); M. Yahiro, M. Kamimura, Prog. Theor. Phys. 65, 2051 (1981); M. Yahiro et al., Prog. Theor. Phys. 67, 1467 (1982).

5. M. Yahiro et al., Prog. Theor. Phys. Suppl. 89, 32 (1986).

6. P. Chau Huu-Tai, Nucl. Phys. A 773, 56 (2006).

7. N.C. Summers, F.M. Nunes, I.J. Thompson, Phys. Rev. C 74, 014606 (2006); F. Skaza et al., Phys. Rev. C 73, 044301 (2006); B. Jonson, Phys. Rep. 389, 1 (2004); J.S. Al-Khalili, F.M. Nunes, J. Phys. G 29, R89 (2003); V. Lapoux et al., Phys. Rev. C 66, 034608 (2002); T. Nakamura et al., Phys. Rev. Lett. 83, 001112 (1999); J.H. Kelley et al., Phys. Rev. Lett. 74, 30 (1995); N.A. Orr et al., Phys. Rev. C 51, 3116 (1995); V. Maddalena et al., Phys. Rev. C 63, 024613 (2001).

8. T. Tamura, Rev. Mod. Phys. 37, 679 (1965).

9. D.A. Varshalovich, A.N. Moskalev, V.K. Khersonskii, Quantum Theory of Angular Momentum (World Scientific, Singapore, 1988)

10. C. Baümer et al., Phys. Rev. C 63, 037601 (2001).

11. A. Korff et al., Phys. Rev. C 70, 067601 (2004).

12. O. Aspelund et al., Nucl. Phys. A 253, 263 (1975).

13. F. Hinterberger et al., Nucl. Phys. A 111, 265 (1968).

14. S. Ishida et al., Phys. Lett. B 314, 279 (1993).

15. A. Kiss et al., Nucl. Phys. A 282, 1 (1976). 